\title{
Synthesis of Depsipeptides via Isocyanide-Based Consecutive Bargellini-Passerini Multicomponent Reactions
}

\author{
Hassan Farhida \\ Mohammad Mahdi Rostamia \\ Ahmad Shaabani*a,b (iD) \\ Behrouz Notash ${ }^{c}$ \\ ${ }^{a}$ Faculty of Chemistry, Shahid Beheshti University, G.C., P.O. \\ Box 19396-4716, Tehran, Iran \\ a-shaabani@sbu.ac.ir \\ b Peoples' Friendship University of Russia (RUDN University), \\ 6, Miklukho-Maklaya Street, Moscow, 117198, Russian Federation \\ ' Department of Inorganic Chemistry and Catalysis, \\ Shahid Beheshti University, Evin, Tehran, Iran \\ Published as part of the Virtual Collection in Honor of Prof. Issa Yavari
}
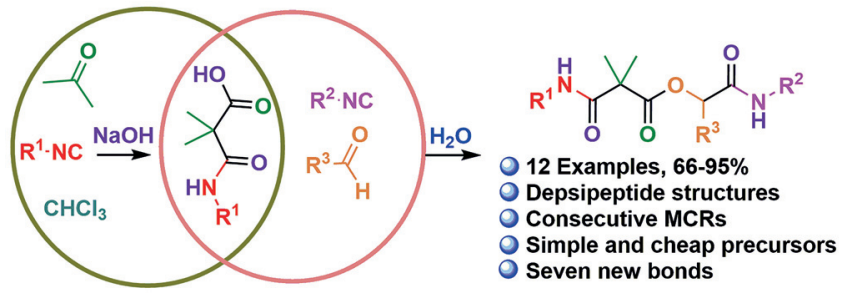

Received: 18.05 .2021

Accepted after revision: 18.06.2021

Published online: 22.06 .2021

DOI: 10.1055/a-1533-3823; Art ID: so-2021-d0027-op

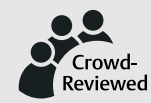

License terms: $c(0)$

(c) 2021. The Author(s). This is an open access article published by Thieme under the terms of the Creative Commons Attribution-NonDerivative-NonCommercial-License, permitting copying and reproduction so long as the original work is given appropriate credit. Contents may not be used for commercial purposes or adapted, remixed, transformed or built upon. (https://creativecommons.org/licenses/by-nc-nd/4.0/)

Abstract An efficient and straightforward approach has been established for the preparation of a new class of depsipeptide structures via isocyanide-based consecutive Bargellini-Passerini multicomponent reactions. 3-Carboxamido-isobutyric acids bearing an amide bond were obtained via Bargellini multicomponent reaction from isocyanides, acetone, and chloroform in the presence of sodium hydroxide. Next, via a Passerini multicomponent-reaction strategy, a new class of depsipeptides was synthesized using the Bargellini reaction products, isocyanides, and aldehydes. The depsipeptides thus prepared have more flexible structures than their pseudopeptidic analogues.

Key words depsipeptide, Passerini reaction, isocyanide

Peptides and proteins accomplish critical functions in numerous biological and physiological operations. In recent years they have received much attention as drug candidates, but their therapeutic applications are limited by low metabolic stability, poor bioavailability, and low receptor affinity. ${ }^{1}$ Consequently, chemists have developed efficient strategies for designing and synthesizing peptidomimetics, which have improved pharmacological properties over their natural peptide analogues. ${ }^{2}$ A significant branch of peptidomimetics is that of the depsipeptides that are generated by replacing one or more amide bonds by an ester functionality. ${ }^{3}$ This modification obviates to some degree intra- and intermolecular hydrogen bonding, which can be advantageous for organ penetration, targeting, and oral bioavailability. ${ }^{4}$ Remarkably, depsipeptides have less rotational barriers for cis-trans isomerization compared to their pep- tide analogues because of reduced resonance delocalization in the ester functionality relative to an amide moiety, and thus have more flexible scaffolds. ${ }^{3,5}$ Furthermore, depsipeptides possess a wide variety of biological properties such as antibacterial, antiviral, antifungal, anti-inflammatory, antitumor, and immunosuppressive activity. ${ }^{6}$ For instance, the natural product romidepsin is an FDA-approved anticancer drug utilized to treat cutaneous T-cell lymphoma. ${ }^{7}$ Other cases of biologically active depsipeptides are azinomycin $\mathrm{B}^{8}$ (antitumor activity) and valinomycin ${ }^{9}$ (antibiotic) that are presented in Figure 1.

Isocyanide-based multicomponent reactions (IMCRs) are highly efficient strategies for peptidomimetic synthesis. ${ }^{10}$ Among the IMCRs, the Ugi four-component reaction has been demonstrated as an extremely powerful approach for the synthesis of pseudopeptidic structures. ${ }^{11}$ The Ugi reaction products are often observed as a mixture of rotamers due to the high rotational barriers of the tertiary amide bonds. ${ }^{12}$ The Passerini three-component reaction (3-CR) that involves an isocyanide, an oxo-component, and a carboxylic acid, is a potent tool in combinatorial chemistry due to its efficiency for producing diverse structures, providing brevity, molecular complexity, and operational simplicity. ${ }^{13,14}$ In general, the Passerini reaction products have more flexible structures than the Ugi reaction products due to possessing an ester functionality instead of the tertiary amide. This is very significant from a drug design viewpoint, because creating faster rotation around a hindered bond is one of the best strategies to overcome the challenge of conformational isomerism. ${ }^{15}$

The scope of MCRs, specially IMCRs, can be further advanced by combination with other sequential reactions ${ }^{16}$ and, in this regard, consecutive MCRs can be a superior selection. ${ }^{17}$ In consecutive MCRs, the product of the first MCR is utilized as the precursor in the next MCR. Accordingly, the diversity, efficiency, and atom economy that are the 

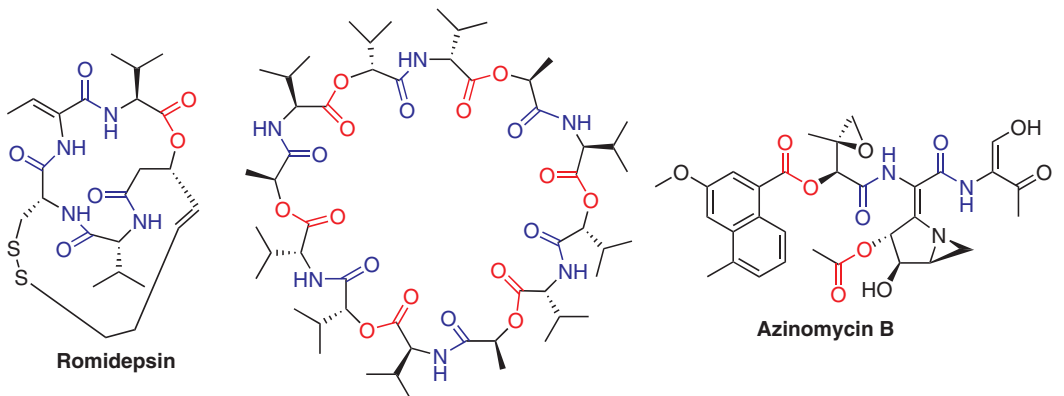

Valinomycin

Figure 1 Some biologically active depsipeptides

benefits of MCRs, are extended. In view of our interests in design of combinatorial MCRs, ${ }^{18}$ we herein report isocyanide-based consecutive Bargellini-Passerini MCRs as a straightforward and efficient strategy for the synthesis of depsipeptide-based frameworks.

In this program, we focused on the using 3-carboxamido-isobutyric acids as the products of Bargellini 3-CRs in the Passerini 3-CRs. 3-Carboxamido-isobutyric acids bearing an amide functionality were synthesized via the 3-CR of acetone, chloroform, and an isocyanide in the presence of sodium hydroxide. ${ }^{19}$ Primarily, compound 4a was employed in the Passerini 3-CR under various reaction conditions (Table 1). The 3-CRs of cyclohexyl isocyanide (3a), benzaldehyde (5a), and compound $\mathbf{4 a}$ were investigated in various solvents such as dichloromethane, toluene, tetrahydrofuran, ethyl acetate, and methanol, and the desired depsipeptide 6a was achieved in moderate yields (24-58\%,
Table 1, entries 1-5). We also examined the model reaction under solvent-free conditions, which led to the depsipeptide $\mathbf{6 a}$ in $61 \%$ yield (Table 1 , entry 6 ). Finally, the reaction was carried out in water as a solvent at ambient temperature and a significant rate enhancement was observed with the production of compound $\mathbf{6 a}$ in $79 \%$ yield (Table 1, entry 7 ). By increasing the reaction temperature to $50{ }^{\circ} \mathrm{C}$, depsipeptide $6 \mathbf{a}$ was produced in $90 \%$ yield after $2 \mathrm{~h}$ (Table 1, entry 8). It is noteworthy that the use of water not only speeds up the MCR, but also makes isolation much easier due to the insolubility of the products. The acceleration of the MCR in water can be related to the hydrophobic effect and the high cohesive energy density of water. ${ }^{20}$ Cohesive energy density is a property of solvents and has units corresponding to a pressure. ${ }^{21}$ The effect of water on the acceleration of MCRs is very similar to the effect of pressure on reactions that result in a decrease in molecularity.

Table 1 Optimization of the Second-Step Reaction Conditions ${ }^{\mathrm{a}}$
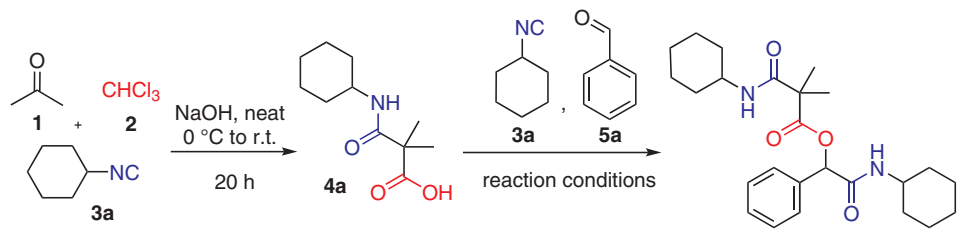

\begin{tabular}{|c|c|c|c|c|}
\hline Entry & Solvent & $\operatorname{Temp}\left({ }^{\circ} \mathrm{C}\right)$ & Time (h) & Yield $(\%)^{b}$ \\
\hline 1 & $\mathrm{CH}_{2} \mathrm{Cl}_{2}$ & r.t. & 24 & 56 \\
\hline 2 & toluene & r.t. & 24 & 48 \\
\hline 3 & THF & r.t. & 24 & 58 \\
\hline 4 & EtOAc & r.t. & 24 & 24 \\
\hline 5 & $\mathrm{MeOH}$ & r.t. & 24 & 36 \\
\hline 6 & - & 80 & 18 & 61 \\
\hline 7 & $\mathrm{H}_{2} \mathrm{O}$ & r.t. & 5 & 79 \\
\hline 8 & $\mathrm{H}_{2} \mathrm{O}$ & 50 & 2 & 90 \\
\hline
\end{tabular}

a Reaction conditions: $\mathbf{3 a}(1 \mathrm{mmol}), \mathbf{4 a}(1 \mathrm{mmol}), \mathbf{5 a}(1 \mathrm{mmol})$, solvent $(4 \mathrm{~mL})$.

b Isolated yield. 
With the optimized reaction conditions in hand, we synthesized a series of depsipeptides $\mathbf{6 a - 1}$ in high yields (Scheme 1). Various aromatic aldehydes with electrondonating, electron-withdrawing, and halogen groups and isocyanides (tert-butyl, cyclohexyl) were employed for demonstrating the diversity of approach. 3-Pyridinecarbaldehyde, 2-naphthaldehyde, and aromatic aldehydes with electron-withdrawing and halogen substitutions afforded the products in excellent yields. 4-Methylbenzaldehyde reacted more slowly in the final Passerini step, requiring $48 \mathrm{~h}$ to achieve a relatively poor $66 \%$ yield. The highly electronrich 4-methoxybenzaldehyde failed to produce any product even after $48 \mathrm{~h}$.

The structures of all depsipeptides $\mathbf{6 a - 1}$ were verified by their IR, ${ }^{1} \mathrm{H}$ NMR, ${ }^{13} \mathrm{C}$ NMR, mass spectra, and $\mathrm{CHN}$ analysis data. For instance, the ${ }^{1} \mathrm{H}$ NMR spectrum of depsipeptide $\mathbf{6 a}$ in $\mathrm{CDCl}_{3}$ as a solvent demonstrated a multiplet for the aromatic protons $(\delta=7.43-7.39 \mathrm{ppm}, 5 \mathrm{H})$, two broad singlets for the NH groups ( $\delta=6.90$ and $6.40 \mathrm{ppm}, 2 \mathrm{H}$ ), a singlet for the benzylic $\mathrm{CH}(\delta=6.06 \mathrm{ppm}, 1 \mathrm{H})$, a broad singlet for the $\mathrm{NH}-\mathrm{CH}$ of the cyclohexyl rings $(\delta=3.80 \mathrm{ppm}, 2 \mathrm{H})$, and a multiplet for the aliphatic protons $(\delta=1.92-1.17 \mathrm{ppm}, 26$ $\mathrm{H})$. The ${ }^{1} \mathrm{H}$-decoupled ${ }^{13} \mathrm{C}$ NMR spectrum of $\mathbf{6 a}$ exhibited three carbonyl groups corresponding to the ester and amide functionalities. The mass spectra of the products showed molecular ion peaks at the accurate $m / z$ values. It is noteworthy that the ${ }^{1} \mathrm{H}$ NMR spectra of the depsipetides $6 \mathbf{a}-\mathbf{g}$ displayed one singlet peak for the benzylic $\mathrm{CH}$ group in both $\mathrm{CDCl}_{3}$ and DMSO- $d_{6}$. Moreover, all the ${ }^{1} \mathrm{H}$-decoupled ${ }^{13} \mathrm{C}$ NMR spectra showed the presence of three carbonyl groups for the depsipeptide scaffolds, which also confirmed the lower rotational barriers for these depsipeptides compared to their corresponding pseudopeptidic structures. ${ }^{18 \mathrm{~d}}$ In addition, the effect of temperature on compound $\mathbf{6 a}$ was investigated at $24-80{ }^{\circ} \mathrm{C}$ using variable-temperature ${ }^{1} \mathrm{H}$ NMR spectroscopy. As shown in Figure 2, no significant changes (except in the chemical shifts of exchangeable $\mathrm{NH}$ groups) were observed with increasing temperature in the spectra. The full width at half maximum for the benzylic $\mathrm{CH}$ group peak is almost constant at investigated temperatures. Therefore, the prepared depsipeptides are demonstrated to have more flexible structures than their pseudopeptidic analogues.

Finally, the structure of $\mathbf{6 a}$ was unambiguously verified by single-crystal X-ray analysis, which is presented in Figure 3 (for detailed information, see the Supporting Information).

$$
\underset{1}{\stackrel{1}{\mathrm{R}^{1}-\mathrm{NC}}+{ }_{3}^{\mathrm{CHCl}_{3}}} \stackrel{\substack{\mathrm{NaOH} \text {, neat } \\ 0^{\circ} \mathrm{C} \text { to r.t. }}}{20 \mathrm{~h}}
$$<smiles>CC(C)(C(=O)NC1CCCCC1)C(=O)OC(C(=O)NC1CCCCC1)c1ccccc1</smiles>

6a, $90 \%$<smiles>Cc1ccc(C(OC(=O)C(C)(C)C(=O)NC2CCCCC2)C(=O)NC2CCC3CCC2C3)cc1</smiles>

$6 e, 66 \%$

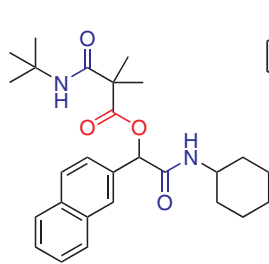

$6 i, 90 \%$

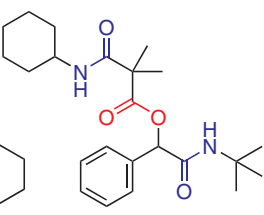

$6 b, 91 \%$<smiles>CC(C)(C)NC(=O)C(C)(C)C(=O)OC(C(=O)NC1CCCCC1)c1ccc(Br)cc1</smiles>

$6 c, 87 \%$

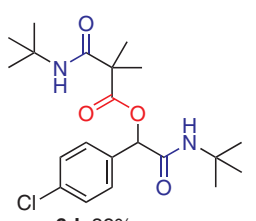

$6 d, 89 \%$<smiles>CCCc1cccc([N+](=O)[O-])c1C(OC(=O)C(C)(C)C(=O)NC(C)(C)C)C(=O)NC(C)(C)C</smiles>

$6 g, 85 \%$

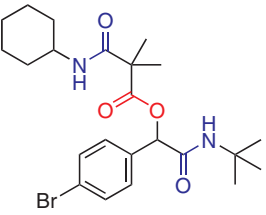

6h, $88 \%$<smiles>CC(C)(C)NC(=O)C(OC(=O)C(C)(C)C(=O)NC1CCCCC1)c1ccc2ccccc2c1</smiles>

$6 \mathbf{6}, 89 \%$

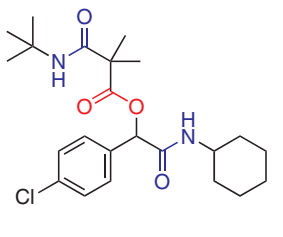

$61,88 \%$

Scheme 1 Scope and yields of the synthesized depsipeptides $\mathbf{6 a - I . ~ R e a c t i o n ~ c o n d i t i o n s ~ o f ~ t h e ~ s e c o n d - s t e p ~ r e a c t i o n : ~ 3 - c a r b o x a m i d o - i s o b u t y r i c ~ a c i d ~} 4$ $(1 \mathrm{mmol})$, aldehyde $5(1 \mathrm{mmol})$, isocyanide $3(1 \mathrm{mmol})$, water $(4 \mathrm{~mL})$, stirring at r.t. for $5 \mathrm{~min}$ and then at $50{ }^{\circ} \mathrm{C}$ for $2 \mathrm{~h}$. Reaction time for $6 \mathbf{e}$ is $48 \mathrm{~h}$. 


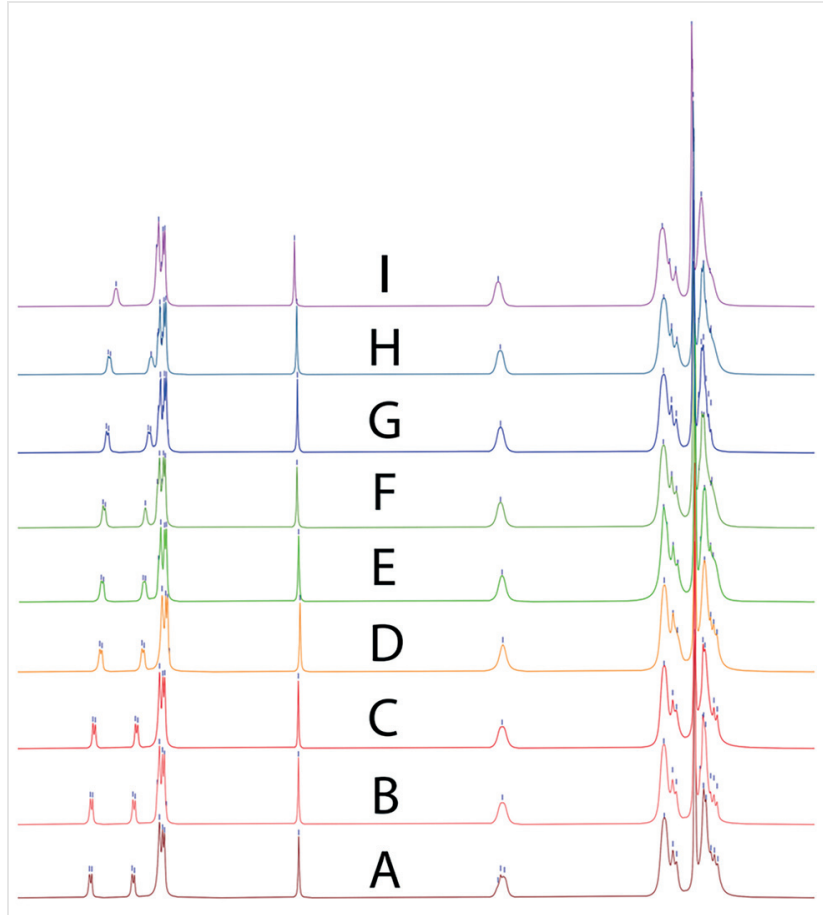

$\begin{array}{llllllllllllllllllllll}1.0 & 8.5 & 8.0 & 7.5 & 7.0 & 6.5 & 6.0 & 5.5 & 5.0 & 4.5 & 4.0 & 3.5 & 1.0 & 2.5 & 2.0 & 1.5 & 1.0 & 0.5 & 0\end{array}$

Figure 2 The ${ }^{1} \mathrm{H}$ NMR spectra of compound $\mathbf{6 a}$ at $24-80^{\circ} \mathrm{C}$ (DMSO- $d_{6}$, $300 \mathrm{MHz}$ ); spectrum A: at $24^{\circ} \mathrm{C}$, spectrum B: at $25^{\circ} \mathrm{C}$, spectrum C: at $30^{\circ} \mathrm{C}$, spectrum $\mathrm{D}$ : at $40^{\circ} \mathrm{C}$, spectrum $\mathrm{E}$ : at $45^{\circ} \mathrm{C}$, spectrum $\mathrm{F}$ : at $50^{\circ} \mathrm{C}$, spectrum G: at $55^{\circ} \mathrm{C}$, spectrum $\mathrm{H}$ : at $60^{\circ} \mathrm{C}$, spectrum I: at $80^{\circ} \mathrm{C}$.

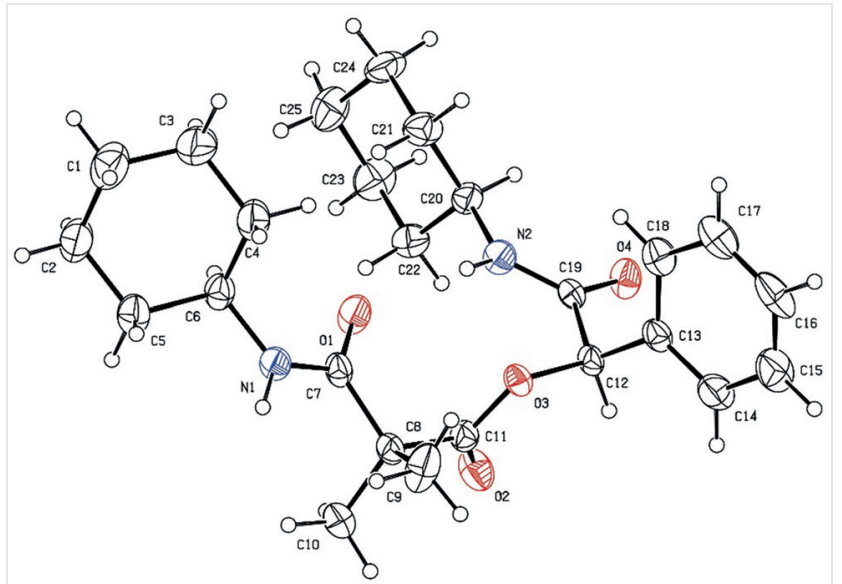

Figure 3 ORTEP diagram for $\mathbf{6 a}$

In summary, a convenient and efficient method has been developed for the preparation of biologically interesting depsipeptides via consecutive Bargellini-Passerini multicomponent reactions. The present method, having the advantages of both IMCRs, leads to the ready creation of depsipeptide scaffolds from cheap and readily available starting materials. The synthesized depsipeptides have more flexible scaffolds than their pseudopeptidic analogues.

\section{General Information}

All commercially available chemicals and reagents were purchased from Merck Chemical Company and used without further purification. Melting points were measured on an Electrothermal 9200 apparatus. IR spectra were recorded on a Shimadzu IR-470 spectrometer. ${ }^{1} \mathrm{H}$ NMR spectra were recorded on a Bruker DRX-300 Avance spectrometer at $300 \mathrm{MHz} .{ }^{13} \mathrm{C}$ NMR spectra were recorded on a Bruker DRX-300 Avance spectrometer at $75 \mathrm{MHz}$. NMR spectra were obtained in $\mathrm{CDCl}_{3}$ and DMSO- $d_{6}$. Mass spectra of the products were obtained with an HP (Agilent technologies) 5973 mass selective detector. Elemental analyses were performed on an Elementar Analysensysteme GmbH VarioEL.

\section{General Procedure for the Synthesis of 3-Carboxamido-isobutyric Acids}

In a round-bottom flask, acetone $(5 \mathrm{mmol})$, chloroform $(7.5 \mathrm{mmol})$, sodium hydroxide $(7.5 \mathrm{mmol})$, and the requisite isocyanide $(1 \mathrm{mmol})$ were mixed and stirred at $0{ }^{\circ} \mathrm{C}$ for $30 \mathrm{~min}$ and then at ambient temperature overnight. The reaction mixture was diluted with water, which was acidified to $\mathrm{pH} 2$ with $2 \mathrm{M} \mathrm{HCl}$ and extracted with EtOAc (three times). The organic layers were washed with brine, dried over $\mathrm{Na}_{2} \mathrm{SO}_{4}$, filtered, and concentrated under reduced pressure to afford the pure product. ${ }^{19 a}$

\section{General Procedure for the Synthesis of Depsipeptides 6a-1}

A mixture of the 3-carboxamido-isobutyric acid derivative (1 mmol), an aldehyde $(1 \mathrm{mmol})$, and an isocyanide $(1 \mathrm{mmol})$ in water $(4 \mathrm{~mL})$ was stirred at room temperature for $5 \mathrm{~min}$ and then at $50{ }^{\circ} \mathrm{C}$ for the appropriate time (Scheme 1). The water was then removed by decantation, and the crude product was recrystallized from EtOH $(2 \mathrm{~mL})$ to afford the pure product.

\section{Characterization Data of 6a-1}

2-(Cyclohexylamino)-2-oxo-1-phenylethyl 3-(cyclohexylamino)2,2-dimethyl-3-oxopropanoate (6a)

White powder: $385 \mathrm{mg}$, 90\% yield; $\mathrm{mp} 145-147{ }^{\circ} \mathrm{C}$. IR (KBr): 3265 , 3087, 2931, 2852, 1745, 1676, $1641 \mathrm{~cm}^{-1} .{ }^{1} \mathrm{H}$ NMR (300 MHz, $\left.\mathrm{CDCl}_{3}\right)$ : $\delta=7.43-7.39\left(\mathrm{~m}, 5 \mathrm{H}, \mathrm{H}_{\mathrm{Ar}}\right), 6.90($ br s, $1 \mathrm{H}, \mathrm{NH}), 6.40($ br s, $1 \mathrm{H}, \mathrm{NH})$, 6.06 (s, $1 \mathrm{H}, \mathrm{CH}-\mathrm{O}$ ), 3.80 (br s, $2 \mathrm{H}, \mathrm{CHN}), 1.92-1.17$ (m, $26 \mathrm{H}, \mathrm{H}_{\text {Aliphatic }}$ ). ${ }^{13} \mathrm{C}$ NMR $\left(75 \mathrm{MHz}, \mathrm{CDCl}_{3}\right): \delta=172.54,171.98,167.51,135.40,128.92$, 128.72, 127.50, 75.96, 50.62, 48.83, 48.51, 32.77, 32.65, 32.58, 25.49, 24.89, 23.33. MS: $m / z=429\left[\mathrm{M}^{+}+1\right](2.52), 428\left(\mathrm{M}^{+}, 0.92\right), 347$ (41.69), 303 (86.77), 234 (7.85), 216 (15.15), 188 (61.60), 169 (100), 140 (10.33), 118 (10.82), 98 (26.33), 83 (30.12), 55 (10.81). Anal. Calcd for $\mathrm{C}_{25} \mathrm{H}_{36} \mathrm{~N}_{2} \mathrm{O}_{4}$ : C, 70.06; H, 8.47; N, 6.54. Found: C, 70.31; H, 8.59; $\mathrm{N}, 6.44$.

2-(tert-Butylamino)-2-oxo-1-phenylethyl 3-(cyclohexylamino)2,2-dimethyl-3-oxopropanoate (6b)

White powder: $366 \mathrm{mg}, 91 \%$ yield; $\mathrm{mp} 173-175{ }^{\circ} \mathrm{C}$. IR (KBr): 3284 , 3080, 2931, 2865, 1741, 1662, $1637 \mathrm{~cm}^{-1} .{ }^{1} \mathrm{H}$ NMR (300 MHz, CDCl $)$ : $\delta=7.39-7.28\left(\mathrm{~m}, 5 \mathrm{H}, \mathrm{H}_{\mathrm{Ar}}\right), 6.62(\mathrm{br} \mathrm{s}, 1 \mathrm{H}, \mathrm{NH}), 6.33(\mathrm{br} \mathrm{s}, 1 \mathrm{H}, \mathrm{NH})$, $5.96(\mathrm{~s}, 1 \mathrm{H}, \mathrm{CH}-\mathrm{O}), 3.79-3.75(\mathrm{~m}, 1 \mathrm{H}, \mathrm{CHN}), 1.90-1.12(\mathrm{~m}, 25 \mathrm{H}$, $\left.\mathrm{H}_{\text {Aliphatic }}\right) .{ }^{13} \mathrm{C}$ NMR $\left(75 \mathrm{MHz}, \mathrm{CDCl}_{3}\right): \delta=172.79,171.56,167.59$, 135.43, 128.99, 128.82, 127.52, 76.04, 51.77, 50.57, 48.78, 32.70, 28.59, 25.53, 24.91, 23.51, 23.38. MS: $m / z=403\left[\mathrm{M}^{+}+1\right]$ (3.56), 402 $\left[\mathrm{M}^{+}\right]$(1.54), 321 (52.20), 303 (89.34), 277 (18.59), 212(7.54), 196 (36.77), 169 (100), 140 (13.69), 106 (46.66), 83 (19.57), 57 (20.78). Anal. Calcd for $\mathrm{C}_{23} \mathrm{H}_{34} \mathrm{~N}_{2} \mathrm{O}_{4}$ : C, 68.63; H, 8.51; N, 6.96. Found: C, 68.78; $\mathrm{H}, 8.43 ; \mathrm{N}, 6.81$. 
1-(4-Bromophenyl)-2-(cyclohexylamino)-2-oxoethyl 3-(tertbutylamino)-2,2-dimethyl-3-oxopropanoate (6c)

White powder: $419 \mathrm{mg}, 87 \%$ yield; mp $133-135{ }^{\circ} \mathrm{C}$. IR (KBr): 3265 , 3080, 2935, 2854, 1749, 1651, $1560 \mathrm{~cm}^{-1}$. ${ }^{1} \mathrm{H}$ NMR (300 MHz, $\left.\mathrm{CDCl}_{3}\right)$ : $\delta=7.50\left(\mathrm{~d}, J=8.1 \mathrm{~Hz}, 2 \mathrm{H}, \mathrm{H}_{\mathrm{Ar}}\right), 7.32-7.29\left(\mathrm{~m}, 3 \mathrm{H}, \mathrm{H}_{\mathrm{Ar}}\right.$ and $\left.\mathrm{NH}\right), 6.02(\mathrm{~s}$, $1 \mathrm{H}, \mathrm{CHO}$ ), 5.80 (br s, $1 \mathrm{H}, \mathrm{NH}$ ), 3.77-3.73 (m, $1 \mathrm{H}, \mathrm{CHN}), 1.94-1.18$ (m, $\left.25 \mathrm{H}, \mathrm{H}_{\text {Aliphatic }}\right) .{ }^{13} \mathrm{C}$ NMR $\left(75 \mathrm{MHz}, \mathrm{CDCl}_{3}\right): \delta=172.40,172.11,166.92$, $134.71,131.79,129.15,122.96,75.20,51.79,51.23,48.59,32.75$, 32.56, 28.58, 25.50, 24.99, 24.92, 23.32. MS: $m / z=482\left[\mathrm{M}^{+}+2\right](1.18)$, $481\left[\mathrm{M}^{+}+1\right]$ (3.14), 467 (7.41), 383 (18.51), 357 (87.60), 294 (16.43), 268 (50.11), 186 (23.68), 170 (37.50), 143 (100), 114 (37.00), 87 (83.60), 57 (44.81). Anal. Calcd for $\mathrm{C}_{23} \mathrm{H}_{33} \mathrm{BrN}_{2} \mathrm{O}_{4}$ : C, 57.38; $\mathrm{H}, 6.91 ; \mathrm{N}$, 5.82. Found: C, 57.51; $\mathrm{H}, 6.80 ; \mathrm{N}, 5.69$.

2-(tert-Butylamino)-1-(4-chlorophenyl)-2-oxoethyl 3-(tert-butylamino)-2,2-dimethyl-3-oxopropanoate (6d)

White powder: $366 \mathrm{mg}, 89 \%$ yield; mp $149-151{ }^{\circ} \mathrm{C}$. IR (KBr): 3296 , 3074, 2972, 2931, 1745, 1664, $1647 \mathrm{~cm}^{-1} .{ }^{1} \mathrm{H}$ NMR (300 MHz, $\left.\mathrm{CDCl}_{3}\right)$ : $\delta=7.35-7.28\left(\mathrm{~m}, 4 \mathrm{H}, \mathrm{H}_{\mathrm{Ar}}\right), 6.88(\mathrm{br} \mathrm{s}, 1 \mathrm{H}, \mathrm{NH}), 5.94(\mathrm{~s}, 1 \mathrm{H}, \mathrm{CHO}), 5.91$ (br s, $1 \mathrm{H}, \mathrm{NH}), 1.47-1.37$ (m, $\left.24 \mathrm{H}, \mathrm{H}_{\text {Aliphatic }}\right) .{ }^{13} \mathrm{C}$ NMR $(75 \mathrm{MHz}$, $\left.\mathrm{CDCl}_{3}\right): \delta=172.33,171.96,167.17,134.78,134.31,128.91,128.84$, 75.31, 51.78, 51.70, 51.15, 28.58, 28.54, 23.43, 23.37. MS: $m / z=412$ $\left[\mathrm{M}^{+}+2\right](0.62), 411\left[\mathrm{M}^{+}+1\right]$ (2.25), 311 (93.36), 255 (7.26), 224 (14.92), 196 (67.58), 170 (29.77), 143 (100), 114 (18.80), 87 (46.69), 57 (39.92). Anal. Calcd for $\mathrm{C}_{21} \mathrm{H}_{31} \mathrm{ClN}_{2} \mathrm{O}_{4}$ : C, 61.38; H, 7.60; N, 6.82. Found: C, 61.51; H, 7.73; N, 6.67.

2-(Cyclohexylamino)-2-oxo-1-(p-tolyl)ethyl 3-(cyclohexylamino)2,2-dimethyl-3-oxopropanoate (6e)

White powder: $292 \mathrm{mg}, 66 \%$ yield; mp $132-134{ }^{\circ} \mathrm{C}$. IR (KBr): 3253 , 3078, 2933, 2854, 1743, 1668, $1635 \mathrm{~cm}^{-1}$. ${ }^{1} \mathrm{H}$ NMR (300 MHz, $\mathrm{CDCl}_{3}$ ): $\delta=7.29-7.14\left(\mathrm{~m}, 4 \mathrm{H}, \mathrm{H}_{\mathrm{Ar}}\right), 6.79(\mathrm{br} \mathrm{s}, 1 \mathrm{H}, \mathrm{NH}), 6.46(\mathrm{br} \mathrm{s}, 1 \mathrm{H}, \mathrm{NH})$, 5.99 (s, $1 \mathrm{H}, \mathrm{CHO}$ ), 3.79-3.68 ( $\mathrm{m}, 2 \mathrm{H}, \mathrm{CHN}), 2.34$ (s, $\left.3 \mathrm{H}, \mathrm{H}_{\mathrm{Me}-\mathrm{Ar}}\right), 1.87-$ $1.14\left(\mathrm{~m}, 26 \mathrm{H}, \mathrm{H}_{\text {Aliphatic }}\right) .{ }^{13} \mathrm{C} \mathrm{NMR}\left(75 \mathrm{MHz}, \mathrm{CDCl}_{3}\right): \delta=172.64,171.97$, $167.69,138.84,132.37,129.40,127.49,75.85,50.57,48.80,48.46$, $32.71,32.56,25.46,24.87,23.31,21.21$. MS: $m / z=443\left[\mathrm{M}^{+}+1\right](2.99)$, $442\left[\mathrm{M}^{+}\right]$(1.01), 361 (21.02), 317 (93.32), 230 (29.57), 202 (67.44), 169 (100), 140 (13.94), 120 (23.23), 98 (34.03), 83 (38.50), 55 (13.39). Anal. Calcd for $\mathrm{C}_{26} \mathrm{H}_{38} \mathrm{~N}_{2} \mathrm{O}_{4}$ : C, 70.56; $\mathrm{H}, 8.65 ; \mathrm{N}, 6.33$. Found: C, 70.78; $\mathrm{H}, 8.82 ; \mathrm{N}, 6.18$.

\section{2-(tert-Butylamino)-1-(2-nitrophenyl)-2-oxoethyl 3-(tert-butyl-} amino)-2,2-dimethyl-3-oxopropanoate (6f)

Pale yellow powder: $392 \mathrm{mg}, 93 \%$ yield; mp $155-157{ }^{\circ} \mathrm{C}$. IR $(\mathrm{KBr})$ : 3303, 3078, 2974, 2875, 1747, 1685, $1647 \mathrm{~cm}^{-1} .{ }^{1} \mathrm{H}$ NMR $(300 \mathrm{MHz}$, $\left.\mathrm{CDCl}_{3}\right): \delta=7.96\left(\mathrm{~d}, J=8.2 \mathrm{~Hz}, 1 \mathrm{H}, \mathrm{H}_{\mathrm{Ar}}\right), 7.66-7.50\left(\mathrm{~m}, 3 \mathrm{H}, \mathrm{H}_{\mathrm{Ar}}\right), 6.98$ (br s, $1 \mathrm{H}, \mathrm{NH}), 6.54$ (s, $1 \mathrm{H}, \mathrm{CHO}), 6.22$ (br s, $1 \mathrm{H}, \mathrm{NH}), 1.44-1.36(\mathrm{~m}$, $\left.24 \mathrm{H}, \mathrm{H}_{\text {Aliphatic }}\right){ }^{13} \mathrm{C}$ NMR $\left(75 \mathrm{MHz}, \mathrm{CDCl}_{3}\right): \delta=172.52,171.89,166.10$, 148.49, 133.49, 130.37, 130.29, 129.72, 124.91, 71.56, 51.94, 51.71, 51.29, 28.57, 28.54, 23.56, 23.38. MS: $m / z=423\left[\mathrm{M}^{+}+2\right](0.73), 422$ $\left[\mathrm{M}^{+}+1\right]$ (2.90), 406 (25.57), 322 (27.87), 253 (49.63), 188 (19.15), 170 (100), 135 (77.73), 114 (33.31), 84 (25.99), 57 (51.08). Anal. Calcd for $\mathrm{C}_{21} \mathrm{H}_{31} \mathrm{~N}_{3} \mathrm{O}_{6}$ : C, 59.84; H, 7.41; N, 9.97. Found: C, 59.63; H, 7.28; N, 10.16 .

\section{2-(Cyclohexylamino)-2-oxo-1-(pyridin-3-yl)ethyl 3-(cyclohexyl-} amino)-2,2-dimethyl-3-oxopropanoate (6g)

White powder: $365 \mathrm{mg}, 85 \%$ yield; mp $184-186{ }^{\circ} \mathrm{C}$. IR (KBr): 3302 , $3057,2931,2855,1743,1685,1657 \mathrm{~cm}^{-1} .{ }^{1} \mathrm{H}$ NMR $\left(300 \mathrm{MHz}, \mathrm{CDCl}_{3}\right)$ : $\delta=8.72-8.59\left(\mathrm{~m}, 2 \mathrm{H}, \mathrm{H}_{\mathrm{Ar}}\right), 7.81-7.77\left(\mathrm{~m}, 1 \mathrm{H}, \mathrm{H}_{\mathrm{Ar}}\right), 7.39-7.29(\mathrm{~m}, 2 \mathrm{H}$, $\mathrm{H}_{\mathrm{Ar}}$ and $\left.\mathrm{NH}\right), 6.14($ br s, $1 \mathrm{H}, \mathrm{NH}), 6.12(\mathrm{~s}, 1 \mathrm{H}, \mathrm{CHO}), 3.83-3.75(\mathrm{~m}, 1 \mathrm{H}$,
CHN), 1.93-1.17 (m, $\left.26 \mathrm{H}, \mathrm{H}_{\text {Aliphatic }}\right) .{ }^{13} \mathrm{C}$ NMR (75 MHz, $\left.\mathrm{CDCl}_{3}\right): \delta=$ 172.16, 171.99, 166.71, 149.99, 148.49, 135.25, 131.58, 123.50, 73.60, $50.75,48.88,48.68,32.88,32.71,32.52,25.46,24.87,23.27$. MS: $m / z=$ $430\left[\mathrm{M}^{+}+1\right](0.20), 429\left(\mathrm{M}^{+}, 0.62\right), 348$ (20.78), 304 (33.35), 235 (15.58), 196 (18.70), 169 (100), 108 (39.53), 83 (46.61), 55 (50.62). Anal. Calcd for $\mathrm{C}_{24} \mathrm{H}_{35} \mathrm{~N}_{3} \mathrm{O}_{4}$ : C, 67.11; $\mathrm{H}, 8.21 ; \mathrm{N}, 9.78$. Found: C, 67.30; H, 8.15; N, 9.89.

1-(4-Bromophenyl)-2-(tert-butylamino)-2-oxoethyl 3-(cyclohexylamino)-2,2-dimethyl-3-oxopropanoate (6h)

White powder: $424 \mathrm{mg}, 88 \%$ yield; $\mathrm{mp} 168-170{ }^{\circ} \mathrm{C}$. IR (KBr): 3253 , 3078, 2933, 2856, 1745, 1639, $1560 \mathrm{~cm}^{-1} .{ }^{1} \mathrm{H}$ NMR (300 MHz, $\left.\mathrm{CDCl}_{3}\right)$ : $\delta=7.51\left(\mathrm{~d}, J=8.0 \mathrm{~Hz}, 2 \mathrm{H}, \mathrm{H}_{\mathrm{Ar}}\right), 7.29\left(\mathrm{~d}, J=8.0 \mathrm{~Hz}, 2 \mathrm{H}, \mathrm{H}_{\mathrm{Ar}}\right), 6.58(\mathrm{br} \mathrm{s}$, $1 \mathrm{H}, \mathrm{NH}), 6.33-6.30(\mathrm{~m}, 1 \mathrm{H}, \mathrm{NH}), 5.91(\mathrm{~s}, 1 \mathrm{H}, \mathrm{CHO}), 3.81-3.75(\mathrm{~m}, 1$ $\mathrm{H}, \mathrm{CHN}), 1.90-1.16\left(\mathrm{~m}, 25 \mathrm{H}, \mathrm{H}_{\text {Aliphatic }}\right) .{ }^{13} \mathrm{C}$ NMR $\left(75 \mathrm{MHz}, \mathrm{CDCl}_{3}\right): \delta=$ 172.41, 171.55, 167.12, 134.62, 131.93, 129.14, 123.12, 75.29, 51.83, $50.57,48.77,32.78,32.67,28.56,25.48,24.85,23.35,23.27$. MS: $m / z=$ $482\left[\mathrm{M}^{+}+2\right]$ (1.53), $481\left[\mathrm{M}^{+}+1\right]$ (3.61), $480\left[\mathrm{M}^{+}\right](0.72), 401$ (24.73), 383 (90.62), 268 (16.27), 242 (53.65), 196 (62.58), 169 (100), 140 (26.15), 98 (37.81), 83 (44.83), 57 (40.60). Anal. Calcd for $\mathrm{C}_{23} \mathrm{H}_{33} \mathrm{BrN}_{2} \mathrm{O}_{4}$ : C, 57.38; H, 6.91; N, 5.82. Found: C, 57.23; H, 6.75; N, 5.73.

2-(Cyclohexylamino)-1-(naphthalen-2-yl)-2-oxoethyl 3-(tertbutylamino)-2,2-dimethyl-3-oxopropanoate (6i)

White powder: $407 \mathrm{mg}$, 90\% yield; $\mathrm{mp} 178-180{ }^{\circ} \mathrm{C}$. IR (KBr): 3257 , 3080, 2933, 2856, 1745, 1645, $1537 \mathrm{~cm}^{-1} .{ }^{1} \mathrm{H} \mathrm{NMR}\left(300 \mathrm{MHz}, \mathrm{CDCl}_{3}\right)$ : $\delta=7.91-7.83\left(\mathrm{~m}, 4 \mathrm{H}, \mathrm{H}_{\mathrm{Ar}}\right), 7.53-7.49\left(\mathrm{~m}, 3 \mathrm{H}, \mathrm{H}_{\mathrm{Ar}}\right), 7.30-7.18(\mathrm{~m}, 1 \mathrm{H}$, $\mathrm{NH}), 6.23$ (s, $1 \mathrm{H}, \mathrm{CHO}), 5.99$ (br s, $1 \mathrm{H}, \mathrm{NH}), 3.79$ (br s, $1 \mathrm{H}, \mathrm{CHN})$, 1.92-1.19 (m, $\left.25 \mathrm{H}, \mathrm{H}_{\text {Aliphatic }}\right) .{ }^{13} \mathrm{C}$ NMR $\left(75 \mathrm{MHz}, \mathrm{CDCl}_{3}\right): \delta=172.51$, $172.39,167.36,133.42,133.06,132.87,128.63,128.20,127.70$, $127.45,126.59,126.41,124.63,76.21,51.73,51.22,48.59,32.81$, $32.62,28.60,25.51,24.98,24.91,23.44,23.40 . \mathrm{MS}: m / z=453\left[\mathrm{M}^{+}+1\right]$ (0.38), $452\left[\mathrm{M}^{+}\right]$(0.05), 327 (82.67), 266 (17.26), 238 (12.03), 170 (42.50), 143 (100), 127 (13.21), 87 (13.57), 57 (14.45). Anal. Calcd for $\mathrm{C}_{27} \mathrm{H}_{36} \mathrm{~N}_{2} \mathrm{O}_{4}$ : C, 71.65; H, 8.02; N, 6.19. Found: C, 71.84; H, 8.23; N, 6.01 .

2-(Cyclohexylamino)-1-(4-nitrophenyl)-2-oxoethyl 3-(cyclohexylamino)-2,2-dimethyl-3-oxopropanoate (6j)

Pale yellow powder: $450 \mathrm{mg}$, 95\% yield; mp $148--150{ }^{\circ} \mathrm{C}$. IR $(\mathrm{KBr})$ : 3269, 3084, 2931, 2860, 1722, 1637, $1529 \mathrm{~cm}^{-1}$. ${ }^{1} \mathrm{H}$ NMR $(300 \mathrm{MHz}$, $\left.\mathrm{CDCl}_{3}\right): \delta=8.21\left(\mathrm{~d}, J=8.6 \mathrm{~Hz}, 2 \mathrm{H}, \mathrm{H}_{\mathrm{Ar}}\right), 7.62\left(\mathrm{~d}, J=8.6 \mathrm{~Hz}, 2 \mathrm{H}, \mathrm{H}_{\mathrm{Ar}}\right)$, 7.42 (br s, $1 \mathrm{H}, \mathrm{NH}$ ), 6.15 (s, $1 \mathrm{H}, \mathrm{CHO}$ ), 5.95 (br s, $1 \mathrm{H}, \mathrm{NH}$ ), 3.81-3.71 (m, $2 \mathrm{H}, \mathrm{CHN}), 1.92-1.18$ ( $\mathrm{m}, 26 \mathrm{H}, \mathrm{H}_{\text {Aliphatic }}$ ). ${ }^{13} \mathrm{C}$ NMR (75 MHz, CD$\left.\mathrm{Cl}_{3}\right): \delta=172.09,171.63,166.21,147.95,142.46,128.05,123.67,74.52$, 50.71, 48.82, 48.68, 32.84, 32.58, 25.32, 24.74, 23.02. MS: $\mathrm{m} / z=474$ $\left[\mathrm{M}^{+}+1\right](1.86), 473\left[\mathrm{M}^{+}\right](0.34), 392$ (43.27), 348 (88.05), 279 (22.05), 233 (23.97), 196 (19.87), 169 (100), 140 (11.12), 98 (24.68), 83 (36.12), 55 (13.51). Anal. Calcd for $\mathrm{C}_{25} \mathrm{H}_{35} \mathrm{~N}_{3} \mathrm{O}_{6}: \mathrm{C}, 63.41 ; \mathrm{H}, 7.45 ; \mathrm{N}$, 8.87. Found: C, $63.27 ; \mathrm{H}, 7.33 ; \mathrm{N}, 8.98$.

2-(tert-Butylamino)-1-(naphthalen-2-yl)-2-oxoethyl 3-(cyclohexylamino)-2,2-dimethyl-3-oxopropanoate (6k)

White powder: $403 \mathrm{mg}, 89 \%$ yield; $\mathrm{mp} 173-175{ }^{\circ} \mathrm{C}$. IR (KBr): 3276 , 3074, 2929, 2856, 1743, 1661, $1636 \mathrm{~cm}^{-1} .{ }^{1} \mathrm{H} \mathrm{NMR} \mathrm{(300} \mathrm{MHz,} \mathrm{CDCl}_{3}$ ): $\delta=7.89-7.84\left(\mathrm{~m}, 4 \mathrm{H}, \mathrm{H}_{\mathrm{Ar}}\right), 7.54-7.49\left(\mathrm{~m}, 3 \mathrm{H}, \mathrm{H}_{\mathrm{Ar}}\right), 6.68(\mathrm{br} \mathrm{s}, 1 \mathrm{H}$, $\mathrm{NH}), 6.41-6.36(\mathrm{~m}, 1 \mathrm{H}, \mathrm{NH}), 6.14(\mathrm{~s}, 1 \mathrm{H}, \mathrm{CHO}), 3.80$ (br s, $1 \mathrm{H}, \mathrm{CHN})$, 1.95-1.14 (m, $\left.25 \mathrm{H}, \mathrm{H}_{\text {Aliphatic }}\right) .{ }^{13} \mathrm{C}$ NMR $\left(75 \mathrm{MHz}, \mathrm{CDCl}_{3}\right)$ : $\delta=172.86$, $171.60,167.59,133.49,133.08,132.75,128.88,128.20,127.76$, $127.53,126.73,126.53,124.51,76.25,51.87,50.62,48.79,32.68$, 28.61, 25.53, 24.91, 23.55, 23.39. MS: $m / z=454\left[\mathrm{M}^{+}+2\right](0.31), 453$ 
[ $\left.\mathrm{M}^{+}+1\right]$ (0.90), 353 (76.95), 240 (9.21), 212 (19.90), 196 (64.18), 169 (100), 141 (37.57), 126 (7.04), 83 (15.64), 57 (14.97). Anal. Calcd for $\mathrm{C}_{27} \mathrm{H}_{36} \mathrm{~N}_{2} \mathrm{O}_{4}$ : C, 71.65; H, 8.02; N, 6.19. Found: C, 71.88; H, 8.28; N, 6.04 .

\section{1-(4-Chlorophenyl)-2-(cyclohexylamino)-2-oxoethyl 3-(tert-} butylamino)-2,2-dimethyl-3-oxopropanoate (61)

White powder: $385 \mathrm{mg}, 88 \%$ yield; mp $147-149{ }^{\circ} \mathrm{C}$. IR (KBr): 3315 , 3064, 2933, 2854, 1747, 1660, $1545 \mathrm{~cm}^{-1}$. ${ }^{1} \mathrm{H}$ NMR (300 MHz, DMSO$\left.d_{6}\right): \delta=8.31-8.28(\mathrm{~m}, 1 \mathrm{H}, \mathrm{NH}), 7.63-7.46\left(\mathrm{~m}, 4 \mathrm{H}, \mathrm{H}_{\mathrm{Ar}}\right), 7.21(\mathrm{br} \mathrm{s}, 1 \mathrm{H}$, $\mathrm{NH}), 5.86$ (s, $1 \mathrm{H}, \mathrm{CHO}), 3.51$ (br s, $1 \mathrm{H}, \mathrm{CHN}), 1.75-1.09(\mathrm{~m}, 25 \mathrm{H}$, $\left.\mathrm{H}_{\text {Aliphatic }}\right){ }^{13} \mathrm{C}$ NMR $\left(75 \mathrm{MHz}\right.$, DMSO- $\left.d_{6}\right): \delta=173.08,172.31,167.26$, $135.16,133.74,129.42,128.93,75.04,51.30,51.15,48.22,32.62$, $32.40,28.72,25.61,24.81,23.63,23.51$. MS: $m / z=438\left[\mathrm{M}^{+}+2\right](0.90)$, $437\left[\mathrm{M}^{+}+1\right]$ (3.20), 421 (9.24), 337 (25.53), 311 (92.67), 250 (20.69), 222 (65.41), 170 (24.96), 143 (100), 125 (26.01), 87 (49.15), 57 (22.25). Anal. Calcd for $\mathrm{C}_{23} \mathrm{H}_{33} \mathrm{ClN}_{2} \mathrm{O}_{4}$ : C, 63.22; $\mathrm{H}, 7.61 ; \mathrm{N}, 6.41$. Found: C, 63.11; H, 7.70; N, 6.58 .

\section{Conflict of Interest}

The authors declare no conflict of interest.

\section{Funding Information}

This work has been supported by the Research Council of Shahid Beheshti University and the RUDN University Strategic Academic Leadership Program (A. Shaabani).

\section{Supporting Information}

Supporting information for this article is available online at https://doi.org/10.1055/a-1533-3823.

\section{References}

(1) (a) Lenci, E.; Trabocchi, A. Chem. Soc. Rev. 2020, 49, 3262. (b) Vlieghe, P.; Lisowski, V.; Martinez, J.; Khrestchatisky, M. Drug Discov. Today 2010, 15, 40.

(2) Nielsen, P. E. Pseudo-Peptides in Drug Discovery; Wiley-VCH: Weinheim, 2004.

(3) Avan, I.; Hall, C. D.; Katritzky, A. R. Chem. Soc. Rev. 2014, 43, 3575.

(4) (a) Abdelraheem, E. M.; Kurpiewska, K.; Kalinowska-Tłuścik, J.; Dömling, A. J. Org. Chem. 2016, 81, 8789. (b) Bucci, R.; Dapiaggi, F.; Macut, H.; Pieraccini, S.; Sironi, M.; Gelmi, M. L.; Erba, E.; Pellegrino, S. Amino Acids 2020, 52, 15.

(5) Kang, Y. K.; Byun, B. J. J. Phys. Chem. B 2008, 112, 9126.

(6) (a) Ballard, C.; Yu, H.; Wang, B. Curr. Med. Chem. 2002, 9, 471. (b) Sarabia, F.; Chammaa, S.; Ruiz, A. S.; Ortiz, L. M.; Herrera, F. L. Curr. Med. Chem. 2004, 11, 1309. (c) Biswas, S.; Avan, I.; Basak, A. K.; Abo-Dya, N. E.; Asiri, A.; Katritzky, A. R. Amino Acids 2013, $45,159$.

(7) VanderMolen, K. M.; McCulloch, W.; Pearce, C. J.; Oberlies, N. H. J. Antibiot. 2011, 64, 525.

(8) Liu, C.; Kelly, G. T.; Watanabe, C. M. Org. Lett. 2006, 8, 1065.
(9) Jaitzig, J.; Li, J.; Süssmuth, R. D.; Neubauer, P. ACS Synth. Biol. 2014, 3, 432 .

(10) (a) Dömling, A. Chem. Rev. 2006, 106, 17. (b) Wessjohann, L. A.; Rhoden, C. R.; Rivera, D. G.; Vercillo, O. E. Cyclic Peptidomimetics and Pseudopeptides from Multicomponent Reactions, In Synthesis of Heterocycles via Multicomponent Reactions I; Springer: Berlin, Heidelberg, 2010, 199. (c) Khalesi, M.; Halimehjani, A. Z.; Franz, M.; Schmidtmann, M.; Martens, J. Amino Acids 2019, 51, 263.

(11) (a) Pelliccia, S.; Alfano, I. A.; Galli, U.; Novellino, E.; Giustiniano, M.; Tron, G. C. Symmetry 2019, 11, 798. (b) Fouad, M. A.; AbdelHamid, H.; Ayoup, M. S. RSC Adv. 2020, 10, 42644. (c) Farhid, H.; Khodkari, V.; Nazeri, M. T.; Javanbakht, S.; Shaabani, A. Org. Biomol. Chem. 2021, 19, 3318.

(12) (a) Gouge, V.; Jubault, P.; Quirion, J.-C. Tetrahedron Lett. 2004, 45, 773. (b) Szczesniak, P.; Maziarz, E.; Stecko, S.; Furman, B. J. Org. Chem. 2015, 80, 3621. (c) Peshkov, A. A.; Peshkov, V. A.; Pereshivko, O. P.; Van der Eycken, E. V. Tetrahedron 2015, 71, 3863. (d) Nazeri, M. T.; Nowee, A. B.; Shaabani, A. New J. Chem. 2021, 45, 3479. (e) Nazeri, M. T.; Farhid, H.; Mohammadian, R.; Shaabani, A. ACS Comb. Sci. 2020, 22, 361.

(13) Reza, Kazemizadeh. A.; Ramazani, A. Curr. Org. Chem. 2012, 16, 418.

(14) (a) Kumar, B.; Maity, J.; Shankar, B.; Kumar, S.; Prasad, A. K. Carbohydr. Res. 2021, 500, 108236. (b) Gulevich, A. V.; Shpilevaya, I. V.; Nenajdenko, V. G. Eur. J. Org. Chem. 2009, 3801. (c) Rostovskii, N. V.; Koronatov, A. N.; Sakharov, P. A.; Agafonova, A. V.; Novikov, M. S.; Khlebnikov, A. F.; Rogacheva, E. V.; Kraeva, L. A. Org. Biomol. Chem. 2020, 18, 9448. (d) Zarezin, D. P.; Shmatova, O. I.; Nenajdenko, V. G. Org. Biomol. Chem. 2018, 16, 5987.

(15) (a) Al-Horani, R. A.; Desai, U. R. Tetrahedron 2012, 68, 2027. (b) Clayden, J.; Moran, W. J.; Edwards, P. J.; LaPlante, S. R. Angew Chem. Int. Ed. 2009, 48, 6398. (c) Glunz, P. W. Bioorg Med. Chem. Lett. 2018, 28, 53

(16) (a) Bariwal, J.; Kaur, R.; Voskressensky, L. G.; Van der Eycken, E. V. Front. Chem. 2018, 6, 557. (b) Sharma, U. K.; Sharma, N.; Vachhani, D. D.; Van der Eycken, E. V. Chem. Soc. Rev. 2015, 44, 1836. (c) Mohammadkhani, L.; Heravi, M. M. Mol. Diversity 2020, 24, 841 .

(17) (a) Zhi, S.; Ma, X.; Zhang, W. Org. Biomol. Chem. 2019, 17, 7632. (b) Zarganes Tzitzikas, T.; Chandgude, A. L.; Dömling, A. Chem. Rec. 2015, 15, 981. (c) Dömling, A. Curr. Opin. Chem. Biol. 2000, 4, 318. (d) Heublein, N.; Moore, J. S.; Smith, C. D.; Jensen, K. F. RSC Adv. 2014, 4, 63627. (e) Yang, L.; Zhang, Z.; Cheng, B.; You, Y.; Wu, D.; Hong, C. Sci. China Chem. 2015, 58, 1734.

(18) (a) Nazeri, M. T.; Mohammadian, R.; Farhid, H.; Shaabani, A.; Notash, B. Tetrahedron Lett. 2020, 61, 151408. (b) Shaabani, S.; Shaabani, A.; Ng, S. W. ACS Comb. Sci. 2014, 16, 176. (c) Hooshmand, S. E.; Ghadari, R.; Mohammadian, R.; Shaabani, A.; Khavasi, H. R. ChemistrySelect 2019, 4, 11893. (d) Farhid, H.; Nazeri, M. T.; Shaabani, A.; Armaghan, M.; Janiak, C. Amino Acids 2021, 53, 1 .

(19) (a) Giustiniano, M.; Pelliccia, S.; Galli, U.; Amato, J.; Travagin, F.; Novellino, E.; Tron, G. C. J. Org. Chem. 2016, 81, 11467. (b) Serafini, M.; Murgia, I.; Giustiniano, M.; Pirali, T.; Tron, G. C. Molecules 2021, 26, 558

(20) Pirrung, M. C.; Sarma, K. D. J. Am. Chem. Soc. 2004, 126, 444.

(21) Pirrung, M. C.; Sarma, K. D. Tetrahedron 2005, 61, 11456. 